\title{
Familial aggregation of mandibular prognathism
}

\section{April Lee ${ }^{1}$ and Chris Yang ${ }^{1}$}

${ }^{1}$ University of Pittsburgh School of Dental Medicine, Pittsburgh PA, USA

\section{Abstract}

Mandibular prognathism is a hereditary condition where there is an excess growth of the mandible in relation to the maxilla that can be associated with maxillary retrusion, mandibular protrusion, or both.

Skeletal mandibular prognathism is most prevalent in Eastern Asian populations. This paper focuses on a Korean family with skeletal mandibular prognathism that was inherited through three generations. Apparently, neither mandible nor maxilla is retruded in the affected individuals, but there is a concave facial profile. The dentition has a class I occlusion with skeletal mandibular prognathism, and the only way to treat this case would be orthognathic surgery with the help of orthodontic appliances.
Citation: Lee A and Yang C. (2015) Familial aggregation of mandibular prognathism. Dentistry 3000. 1:a001 doi:10.5195/d3000.2015.32

Received: March 20, 2015

Accepted: March 26, 2015

Published: April 8, 2015

Copyright: (C2015 Lee A and Yang C. This is an open access article licensed under a Creative Commons Attribution Work 4.0 United States License.

Email: akl33@pitt.edu

\section{Introduction}

Mandibular prognathism is a complex, hereditary condition where the skeletal profile is concave: the mandible is large or there is underdeveloped or normal maxilla. The dentition can be class I not necessarily class III) in order to have true skeletal mandibular prognathism.

The condition can be presented with distinct cephalometric features such as a short anterior cranial base length, acute cranial base angle, short retrusive maxilla, proclined maxillary incisors, retro-

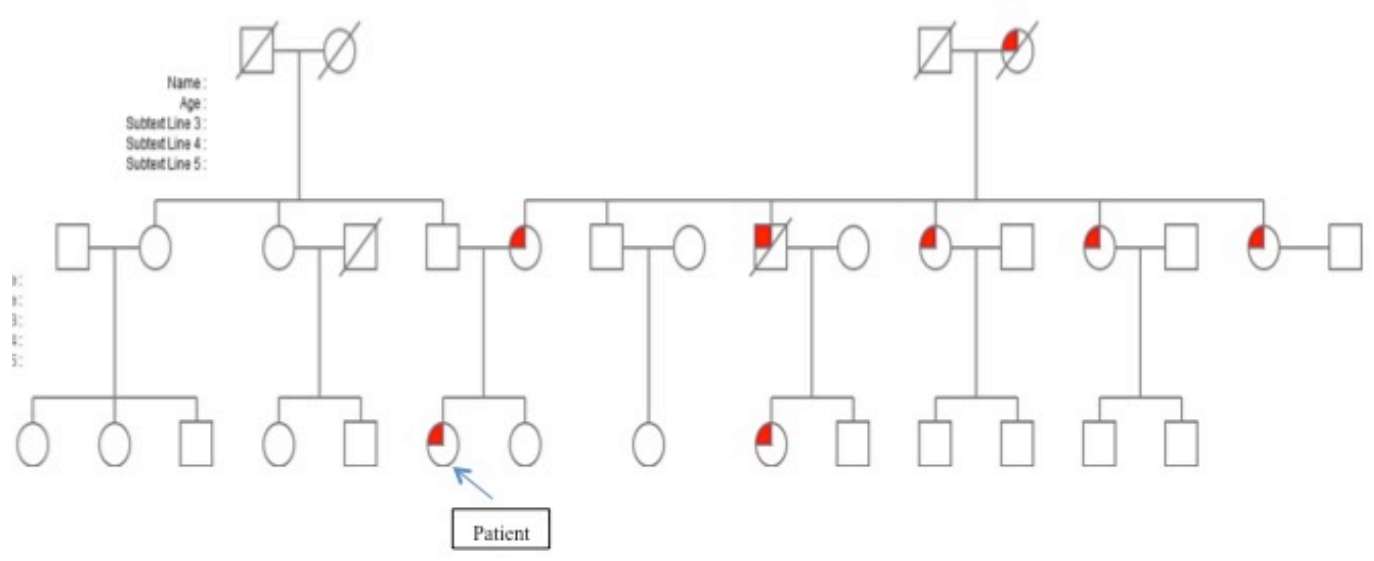

clined mandibular incisors, an excessive lower anterior face height and obtuse gonial angle. It can be associated with maxillary retrusion or mandibular protrusion or both [1].

Mandibular prognathism can be expressed to different degrees based on sex and ethnicity. Even though esthetics is an initial concern for a patient with malocclusion, there can also be functional concerns (i.e., temporomandibular joint dysfunction), and psychological problems that may affect the patient $[2,3]$. In this paper, a family segregating mandibular prognathism is described with the suggestion there is a strong genetic component to this phenotype.

Figure 1. Pedigree of the family.

New articles in this journal are licensed under a Creative Commons Attribution 4.0 United States License.

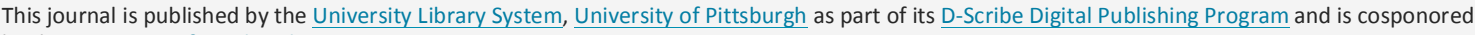
by the University of Pittsburgh Press. 


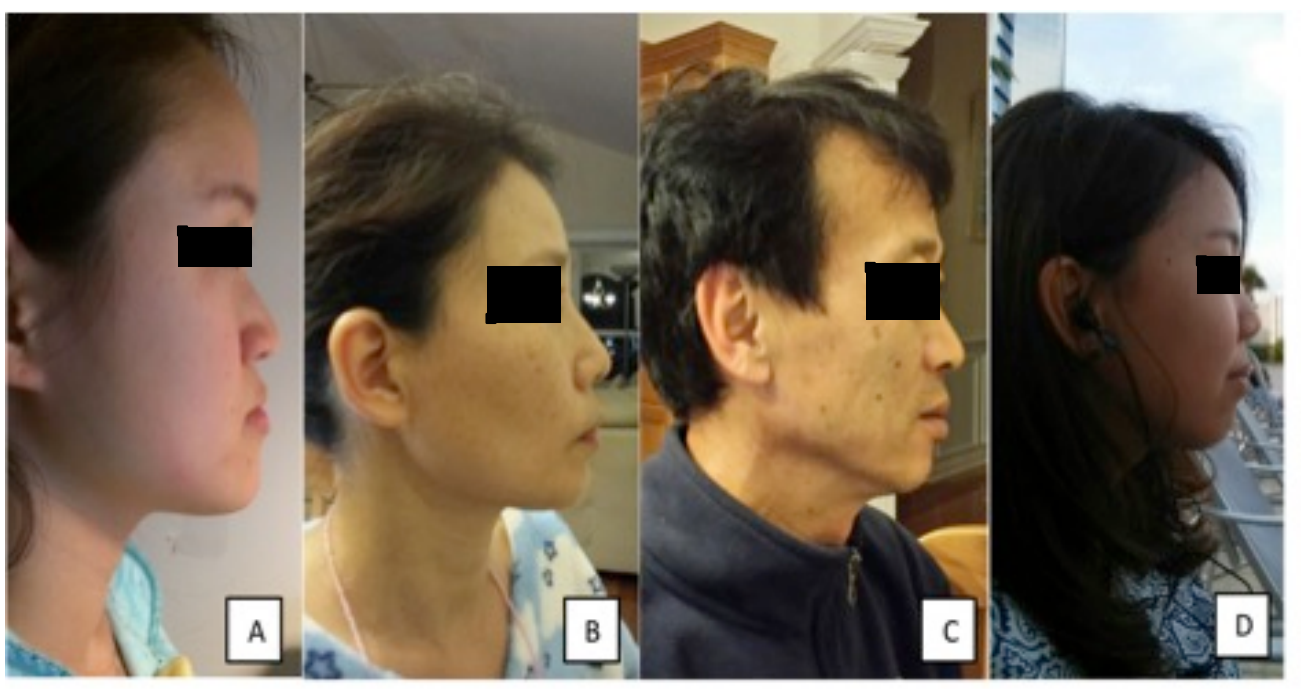

Figure 2. A. A.L (patient) [C.III] B. Mother of A.L [C.III] C. Father of A.L [C.I] D. Sister of A.L [C.I]

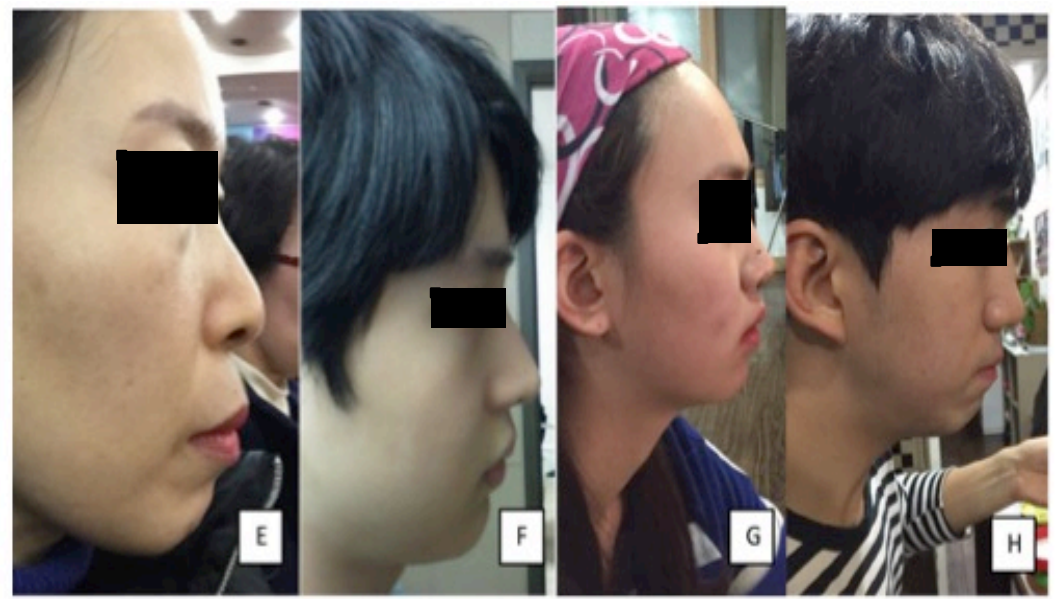

Figure 3.E. Sister of (figure 2B) [C.III] F. Son of (figure 3E) [C.II] G. Figure 2B's deceased brother's daughter [borderline C.III] H. Figure 2B's deceased brother's son [C.I]

\section{Case Report}

This paper describes a 24year-old female who has been concerned about her esthetics with having a projected mandible, giving her a concave profile. Her maxilla is positioned slightly backwards in regards to the mandible. This gives her an appearance of "flat nose" and seemingly smaller cheekbones. She has slight frontal bossing, and consequently her forehead is not in line with the maxilla or mandible, giving her the concave profile.

The patient's mother has mandibular prognathism. The mother's side of the family members has similar profiles: three of

\section{Discussion}

It seems that mandibular prognathism is most prevalent in Eastern Asian population, which can range from $8-40 \%$. There seems to be no significant sexual preference for inheritability, but there seems to be significant difference in sexual dimorphism. There can be five distinct subphenotypes of class III malocclusion: 1) prognathic mandible with long face, 2) maxillary deficiency with decreased vertical dimensions, 3) maxillary deficiency with increased vertical dimensions, 4) mild prognathic

mandible with normal vertical dimensions, and 5) combination of prognathic mandible and maxillary deficiency with normal vertical dimension [1]. of those subphenotypes, this family seems to have mild prognathic mandible with normal vertical dimensions.

According to the literature, manher mother's sisters, one of her mother's brothers, and her grandmother. The mother's sisters all have children who do not have mandibular prognathism, but her brother has one daughter with subtle sign of class III and a son with class I. However, the patient's father and her younger sister both have straight profiles (Figure 1, 2, 3).

The patient was still concerned with her looks, so she was considering getting orthodontics or orthognathic surgery. However, her dentition was class I occlusion with perfectly normal function. Therefore, nothing was performed. dibular prognathism shows autosomal dominance with incomplete penetrance with variable expressivity, or it could be multifactorial. The prevalence varies among different ethnicities, and it can show variable anatomic characteristics [1]. In this family, no one on her father's side was affected with having class III malocclusion, but many individuals on her mother's side of the family were affected. There seems to be no sexual preference in inheritability.

As a dentist, if the patient is concerned with esthetics, the dentist can help the patient to feel more confident by considering orthodontics or orthognathic surgery, but 
s/he should also be able to present the negative aspects of the treatment. If the patient was not concerned with esthetics but the function was an issue, then the dentist should foresee the problem for the patient in the long term and give all the options available for the patient to progress with treatment.

Since the patient had no functional problems with occlusion, the dentist should discuss with patient the pros and cons of orthodontics or orthognathic surgery. The pros would be improving esthetics, but on the downside, it can decrease function, weaken teeth, long recovery period, and create a financial burden for the patient.

\section{References}

1. James K. Hartsfield Jr., Lorri Ann Morford and Liliana M. Otero (2012). Genetic Factors Affecting Facial Growth, Orthodontics - Basic Aspects and Clinical Considerations, Prof. Farid Bourzgui (Ed.), ISBN: 978-953-510143-7, InTech, Available from: http://www.intechopen.com/books/ orthodontics-basic-aspects-andclinicalconsiderations/ genetic-factors-affecting-facialgrowth

2. Orthodontic-surgical treatment of Class III malocclusion with extraction of an impacted canine and multisegmented maxillary surgery. Janson $M$, Janson $G$, Santana $E$, de Castro RC, de Freitas MR. Am J Orthod Dentofacial Orthop. 2010 Jun;137(6):840-9. doi: 10.1016/j.ajodo.2007.12.036.

PMID: 20685541

3. Surgical-orthodontic treatment of a skeletal class III malocclusion. Katiyar
R, Singh G, Mehrotra D, Singh A. Natl J Maxillofac Surg. 2010 Jul-Dec; 1(2):143-149. PMCID: PMC3304204 